\title{
La contabilidad asistida mediante un ERP. Experiencia en el aula
}

\author{
GLORIA SÁNCHEZ-LOZANO \\ Universidad de Sevilla \\ Departamento de Economía Financiera \\ y Dirección de Operaciones \\ gsanchez7@us.es \\ D.0.1.: http://dx.doi.org/10.12795/JDU.2018.i01.87 \\ Pp.: 1551-1569
}

\section{Resumen}

El presente trabajo se centra en explicar el diseño y aplicación de un ciclo de mejora docente en una parte práctica de la asignatura "Sistemas de Información para las Finanzas y la Contabilidad", del Grado de Finanzas y Contabilidad. La experiencia se engloba dentro del marco de trabajo propuesto por la Red de Formación e Innovación Docente (REFID) y tiene lugar durante el curso académico 2018-19 (Primer Cuatrimestre). La evaluación del ciclo se lleva a cabo mediante un cuestionario de aplicación inicial y final en el que se observa una evolución positiva en el aprendizaje de los alumnos con respecto a la mayoría de los conceptos estudiados. Asimismo, el docente evalúa su experiencia de forma reflexiva, detectando los aspectos positivos y pendientes de mejora del ciclo con la intención de aplicarlos en diseños futuros, asegurando asi una mejora progresiva en la calidad docente.

Palabras clave: Organización de empresas, ERP, docencia universitaria, experimentación docente universitaria, innovación docente. 


\section{Breve descripción del contexto}

El ciclo de mejora se ha experimentado en la asignatura de cuarto curso "Sistemas de Información para las Finanzas y la Contabilidad" (SIFICO, en adelante), impartida en el Doble Grado en Derecho y en Finanzas y Contabilidad.

Se trata de una asignatura cuatrimestral de carácter obligatorio ( 6 créditos) donde se ha desarrollado la experiencia en tres sesiones prácticas dedicadas a la Contabilidad utilizando el software de apoyo Aban-Q. La asistencia a esta parte de la asignatura no es obligatoria, aunque en torno al $70 \%$ de los matriculados asiste regularmente. La gran mayoría de los alumnos opta por la evaluación continua para superar esta asignatura, por lo que la predisposición general es bastante favorable. Este año contamos con 34 alumnos matriculados en este grupo.

Los alumnos presentan ciertas reticencias a trabajar con el software, puesto que consideran que hay otros ERPS más actuales y de uso extendido en el mercado de trabajo y, a veces, a trabajar siguiendo un manual de prácticas, el cual constituye el eje central de todas las sesiones aplicadas de la asignatura. El objetivo de usar este manual y de la organización de la asignatura en bloques temáticos muy planificados es poder conseguir cierta estandarización de los contenidos que se imparten a los alumnos de los diez grupos en que se dividen los matriculados en SIFICO, así como facilitar la coordinación entre el número considerable de docentes que intervienen en la asignatura $y$, en ocasiones, en los grupos. Se garantizan unos mínimos deseables, si bien es cierto que la planificación incide de lleno en la metodología que tiene cabida en el aula. Innovar de forma aislada en uno de los grupos puede llegar a ser contraproducente para esos alumnos, puesto que, finalmente, se enfrentarán a la misma evaluación continua o final que los compañeros de otros grupos. 
Las sesiones se imparten en las aulas de informática de la Facultad de Turismo y Finanzas, donde los equipos son actuales. El profesor puede proyectar la pantalla de su ordenador para ir mostrando y resolviendo los casos que plantea el manual.

En los siguientes apartados se explicará el diseño del Ciclo de Mejora Docente, atendiendo a experiencias anteriores, el contenido de las sesiones, la metodología y evaluación prevista del diseño. A continuación del diseño, se comentará la experiencia durante su aplicación y los resultados obtenidos, por último, se reflexionará y evaluará el diseño del ciclo con el objetivo de mantener aquellos aspectos que hayan resultado favorables y proponer posibles mejoras a considerar en futuros diseños.

\section{Diseño previo del Ciclo de Mejora Docente}

\section{Conexión con el proceso previo}

En una ocasión anterior, la docente pudo poner en práctica su primer ciclo de mejora en la misma asignatura, aunque en sesiones dedicadas a la Facturación. No obstante, al tratarse de sesiones prácticas trabajando con el mismo software (en distintos módulos o áreas del programa), se van a poder recoger fácilmente los aspectos positivos que ya dieron sus frutos en la anterior experiencia adaptándolos a los nuevos contenidos.

Se volverá a basar el modelo en ejercicios realistas e interacción con los alumnos mediante soluciones argumentadas entre grupos, ya que la actividad tuvo buena acogida. Se creará y repartirá la "hoja de ruta" de cada sesión, porque favoreció el seguimiento de los alumnos sin necesitar abrir manual, a la vez que no se desatendían sus 
contenidos. Por último, se intentará promover los principios didácticos en los que se basó el anterior ciclo por su ayuda en la creación de un buen ambiente de trabajo y mantenimiento de la motivación.

Como novedad en la propuesta de diseño, el ciclo de mejora se va a desarrollar en un único grupo durante las 6 horas de trabajo, experiencia que resultará menos limitante que la anterior. Ésta última tuvo lugar en tres grupos diferentes durante una única sesión de dos horas, estableciendo la comparativa de experiencias entre grupos. También se reducirá en medida de lo posible el número de casos prácticos incluidos en la secuencia de actividades, ya que uno de los problemas detectados fue la sobre planificación de la sesión. Las interacciones con los grupos de alumnos requieren un tiempo variable y es necesario tener cierto margen entre actividades. Por último, se va a profundizar en el diseño y análisis de los cuestionarios, empleando la técnica de escaleras de aprendizaje a nivel grupal, esta vez mediante preguntas abiertas en lugar de preguntas cerradas de tipo test.

\section{Mapa o red de contenidos y problemas o casos}

En el mapa conceptual que se presenta a continuación (Figura 1, Mapa Conceptual de las sesiones del Ciclo), se intentan enlazar los conceptos clave con las sesiones anteriores dedicadas a la Facturación, comprendiendo cómo el registro de facturas y la gestión de recibos ha ido teniendo sus efectos contables mediante el registro simultáneo de asientos o apuntes que son efecto de la contabilidad integrada que permite un software ERP (Pregunta 1). 


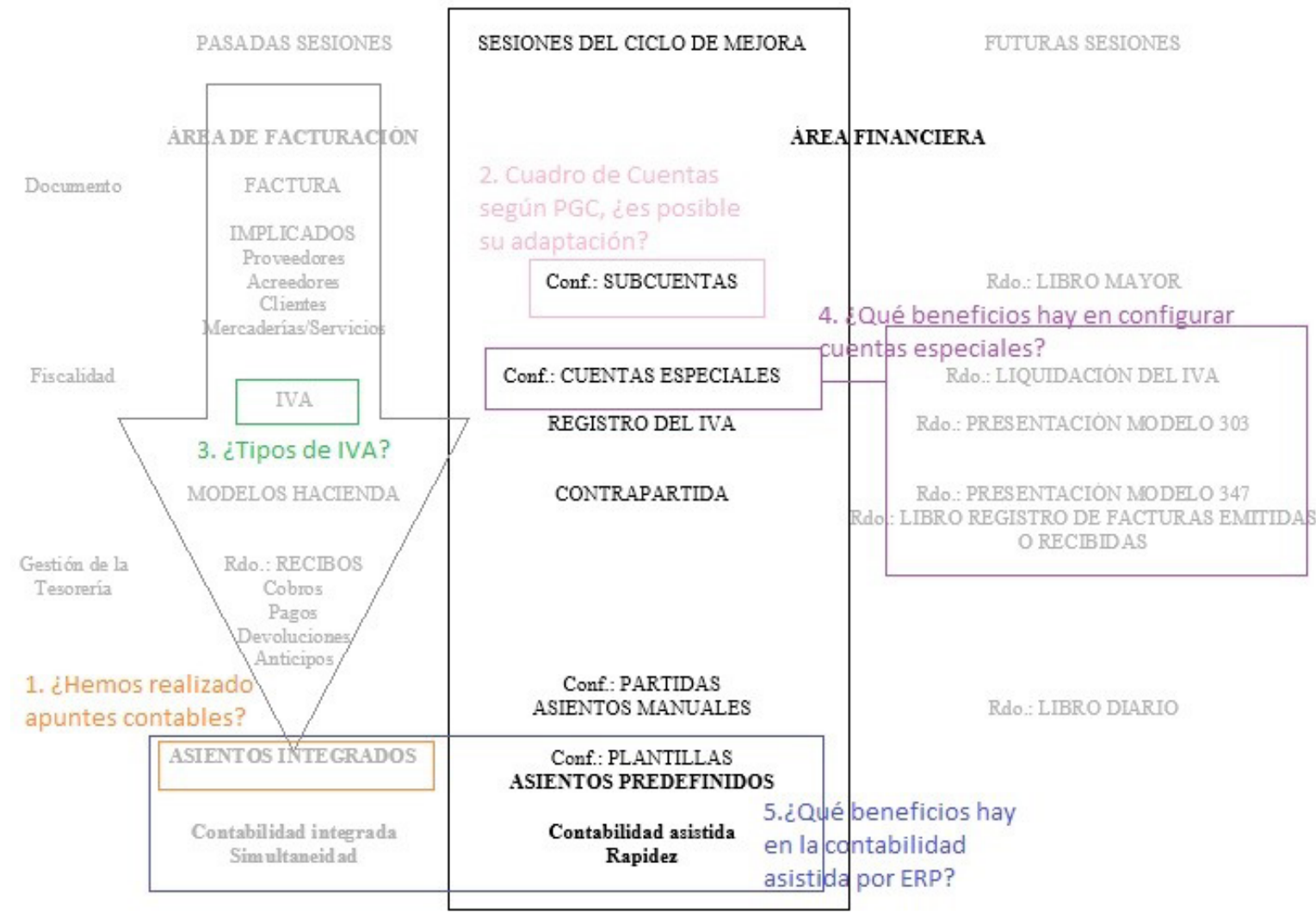

Figura 1. Mapa conceptual de las sesiones del Ciclo.

Jornadas de Formación e Innovación Docente del Profesorado | № 1 (2018)

cc (i) $\ominus$ Esta obra se distribuye con la licencia Creative Commons

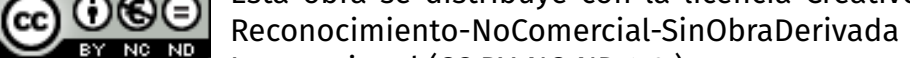

Internacional (CC BY-NC-ND 4.0.) 
Al presentar los contenidos de sesiones posteriores al ciclo, tratamos de conectar con los resultados, modelos e informes que se podrán obtener en sesiones posteriores de Contabilidad. Esto nos ayuda a aportar una utilidad más tangible a las operaciones de configuración necesarias.

Seguidamente, se abordan temas de configuración del Área Financiera desde conceptos ya conocidos. En el momento de registrar las facturas, necesitábamos indicar los proveedores, clientes y artículos que participaban en esa operación. Para ello, estábamos creando subcuentas contables para cada uno de los clientes y proveedores (entre otros) y en las sesiones del ciclo recordaremos que (Pregunta 2) podemos adaptar el Cuadro de Cuentas, a nivel de Subcuentas, a la actividad de nuestra empresa, así como visualizar los cambios de saldos en el Libro Mayor de cada subcuenta contable de proveedor o cliente, entre otros. Alli aparecerán los importes que provienen de las facturas registradas anteriormente y quedará patente de nuevo el efecto de la contabilidad integrada.

Si bien el IVA ya se abordó durante las primeras sesiones de la asignatura, cuando se configuró el menú relativo a Fiscalidad-Impuestos, ahora vuelve a tomar especial protagonismo por la necesidad de registrar facturas exentas de IVA y conocer las implicaciones de este impuesto a la hora de introducir asientos y obtener resultados tan importantes como la Liquidación del IVA y la presentación de Modelos a Hacienda. Se abordan estos temas consultando (Pregunta 3) los tipos de IVA conocidos por los alumnos, contabilizando una operación exenta de IVA (ampliación de conocimiento) y la necesidad, tanto de configurar (Pregunta 4) las Cuentas Especiales como especificar las Contrapartidas para obtener más adelante las liquidaciones y Modelos 303, 309 y 347.

Finalmente, se aborda la posibilidad de programar plantillas para aquellos apuntes contables que sean habituales 
en nuestra empresa, empleando los Asientos Predefinidos. Se presentan sus efectos (Pregunta 5), como una mayor rapidez y productividad de los empleados del Departamento de Contabilidad (reduciendo errores de contabilización, disminuyendo la necesidad de formación contable, agilizando tareas repetitivas) lo que redundará en unos menores costes y rentabilidad de la empresa desde el punto de vista del empresario. Serán los propios alumnos los que seguramente califiquen estos efectos como positivos, al experimentar por ellos mismos, el ahorro de tiempo al contabilizar asientos manuales y predefinidos.

Las preguntas que se detallan y relacionan en el Mapa Conceptual se verán reflejadas, de forma más extensa, en los cuestionarios de evaluación inicial y final.

\section{Modelo Metodológico ideal y posible para el nuevo CMD. Secuencia de actividades.}

Mi modelo metodológico ideal consistiría en llevar documentos reales o ficticios con apariencia real (facturas de venta, de compra, recibos de pagos por arrendamiento de locales, pagos de nóminas de personal, etc.) enfrentando a los alumnos por grupos, a su contabilización utilizando el software que se está explicando. Una vez comenzase la lluvia de preguntas por su parte, navegaría de forma breve por los menús del programa para orientarles acerca de la configuración necesaria para su correcto funcionamiento, hasta acompañarlos a las secciones donde se puede comenzar a registrar los asientos contables de forma manual. Me serviría de algún alumno que esté realizando los apuntes de forma más o menos acertada para explicar cómo lo ha resuelto él y recoger las propuestas de otros compañeros. Una vez hayan probado el arduo trabajo de contabilizar mediante asientos manuales varios documentos, les explicaría las bondades de tomarse un tiempo en configurar los asientos predefinidos, los cuales hacen sus veces 
de "plantillas" reutilizables, ahorran tiempo, esfuerzo y costes a la empresa. Cada grupo se encargaría de comentar cómo ha resuelto un tipo de documento diferente y debatiriamos sobre las diferentes formas de configurar las plantillas y realizar estos apuntes contables.

El modelo metodológico habitual difiere en cierta medida. Está centrado en el uso del manual de clases prácticas, que si bien incluye situaciones que se dan en la empresa, también expone seguidamente la forma correcta de resolverlo con el programa. En ese momento el alumno no presta tanta atención, primero porque ya tiene la respuesta antes de haberle nacido la curiosidad $\mathrm{y}$, en segundo lugar, porque el profesor, si no realiza un esfuerzo por conectar, puede ser percibido como un "reproductor" de las pantallas del manual que actúa en directo.

En el modelo metodológico posible, se han intentado recoger los principios didácticos de Finkel (2005) y Bain (2007) que ya se tuvieron en consideración en el anterior ciclo (Sánchez-Lozano, 2016). En concreto, de Finkel se recogen las ideas de trabajo por grupos de forma reflexiva sobre un conjunto de actividades organizadas y diseñadas por el docente, el cual no ofrece soluciones y correcciones directas, sino que guía y orienta al alumno para que las descubra por sí mismo. El final significativo del proceso de enseñanza-aprendizaje que propone el autor, se materializa en la presentación por grupos de sus propuestas y debate conjunto con otros grupos para mejorarlas. De los principios didácticos de Bain, se propone la entrega de material suplementario ("hoja de ruta de la sesión" en este caso) y la creación de un ambiente distendido y motivador. En concreto, para aumentar la motivación, la secuencia de actividades pretende poner al alumno en contacto con la realidad profesional. Mediante la exposición y debate de sus propuestas de resolución de los casos, se persigue que puedan descubrir en qué medida las aportaciones de su grupo serían valiosas en este futuro contexto laboral. 
La metodología empleada consta de cuatro fases, con participación creciente de los alumnos a medida que avanza la sesión (Figura 2, Metodología posible del CMD). En primer lugar, una narración introductoria donde se enlaza con las sesiones anteriores, se entrega la "hoja de ruta" y se introduce brevemente la estructura de esa sesión; una o varias actividades que pueden ir desde puramente narrativa a narración interactiva con preguntas a los alumnos; por último, se configuran los grupos de trabajo, los alumnos resuelven los ejercicios en común y el profesor sólo orienta a los alumnos para que sean ellos los que solventen sus propias dudas. Al final de la sesión, cada grupo expone su solución a uno de los casos prácticos. Los demás alumnos valoran y complementan las aportaciones de los compañeros.

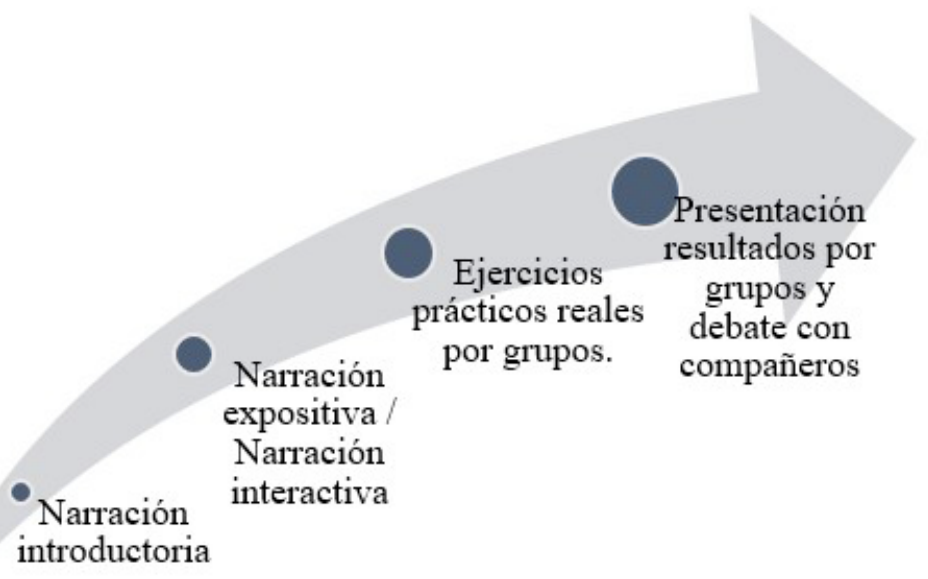

Figura 2. Metodología posible del CMD

La secuencia de actividades programadas para estas sesiones se presentan varias tablas, una para cada sesión del ciclo, junto con los objetivos y tiempos aparejados a cada una de ellas. 
Tabla 1. Secuencia de actividades de la primera sesión

\begin{tabular}{|c|c|c|c|c|}
\hline & $\begin{array}{l}\text { Tipo de } \\
\text { actividad }\end{array}$ & Actividad & Objetivos/Conceptos & $\begin{array}{l}\text { Tiempo } \\
\text { previsto }\end{array}$ \\
\hline 1 & $\begin{array}{l}\text { Narración } \\
\text { introductoria }\end{array}$ & $\begin{array}{l}\text { Resolución de du- } \\
\text { das y breve repaso } \\
\text { de la clase anterior. } \\
\text { Introducción de ac- } \\
\text { tividades a desa- } \\
\text { rrollar durante la } \\
\text { sesión }\end{array}$ & $\begin{array}{l}\text { Conexión con el hilo } \\
\text { de clases anterio- } \\
\text { res y agilidad en los } \\
\text { cambios de tareas } \\
\text { por conocer lo que va } \\
\text { a ocurrir durante la } \\
\text { sesión }\end{array}$ & 10 minutos \\
\hline 2 & $\begin{array}{l}\text { Actividad } \\
\text { exploratoria }\end{array}$ & $\begin{array}{l}\text { Aplicación del cues- } \\
\text { tionario inicial } \\
\end{array}$ & $\begin{array}{l}\text { Evaluación de conoci- } \\
\text { mientos previos. }\end{array}$ & 15 minutos \\
\hline 3 & $\begin{array}{l}\text { Narración- } \\
\text { exposición }\end{array}$ & $\begin{array}{l}\text { Enlazar el nuevo } \\
\text { módulo del sof- } \\
\text { tware con el que se } \\
\text { ha visto en las se- } \\
\text { siones dedicadas a } \\
\text { Facturación }\end{array}$ & $\begin{array}{l}\text { Entender cómo es- } \\
\text { tán integrados los } \\
\text { módulos del sof- } \\
\text { tware viendo el en- } \\
\text { lace de acciones que } \\
\text { ellos mismos han } \\
\text { realizado. }\end{array}$ & 25 minutos \\
\hline 4 & $\begin{array}{l}\text { Exposición } \\
\text { práctica }\end{array}$ & $\begin{array}{l}\text { Realizar las ope- } \\
\text { raciones necesa- } \\
\text { rias para poder } \\
\text { usar el módulo Área } \\
\text { Financiera. }\end{array}$ & $\begin{array}{l}\text { Configurar aspectos } \\
\text { necesarios: } \\
\text {-Cuentas especiales } \\
\text {-Conceptos de } \\
\text { partidas } \\
\text {-Subcuentas }\end{array}$ & 20 minutos \\
\hline 5 & $\begin{array}{l}\text { Ejercicio } \\
\text { práctico con } \\
\text { software }\end{array}$ & Asiento de apertura & $\begin{array}{l}\text { Registrar el asiento } \\
\text { de apertura (sin IVA) } \\
\text { nos ayuda a entender } \\
\text { el proceso de registro } \\
\text { de asientos manuales } \\
\text { en su forma más sim- } \\
\text { plificada, además de } \\
\text { adaptar el cuadro de } \\
\text { cuentas a las necesi- } \\
\text { dades de la empresa } \\
\text { mediante la creación } \\
\text { de subcuentas. }\end{array}$ & 20 minutos \\
\hline
\end{tabular}

La secuencia de actividades se está programando para un tiempo inferior (90 minutos) al de duración de una sesión habitual (120 minutos), contando con posibles 
retrasos en los cambios de actividad, solventar problemas de los equipos y cualquier contratiempo. Además, en cada una de las sesiones hay que restaurar una copia de seguridad al comienzo para poder trabajar, y al final, se debe realizar un resguardo de la copia de seguridad nueva, la cual contiene las modificaciones que se hayan introducido durante la clase. 
Tabla 2. Secuencia de actividades de la segunda sesión.

\begin{tabular}{|c|c|c|c|c|}
\hline & $\begin{array}{l}\text { Tipo de } \\
\text { actividad }\end{array}$ & Actividad & Objetivos/Conceptos & $\begin{array}{l}\text { Tiempo } \\
\text { previsto }\end{array}$ \\
\hline 1 & $\begin{array}{l}\text { Narración } \\
\text { introductoria }\end{array}$ & $\begin{array}{l}\text { Retomamos los } \\
\text { Asientos Manuales } \\
\text { conectando con el } \\
\text { ejercicio de la se- } \\
\text { sión anterior. }\end{array}$ & $\begin{array}{l}\text { Conectar con el tema } \\
\text { que hoy se desarro- } \\
\text { llará más profunda- } \\
\text { mente. Solventar las } \\
\text { dudas que les haya } \\
\text { podido surgir al ter- } \\
\text { minar de registrar el } \\
\text { Asiento de Apertura. }\end{array}$ & $\begin{array}{l}10 \\
\text { minutos }\end{array}$ \\
\hline 2 & $\begin{array}{l}\text { Ejercicio } \\
\text { práctico con } \\
\text { software }\end{array}$ & $\begin{array}{l}\text { Asiento manual por } \\
\text { la adquisición de } \\
\text { un ordenador me- } \\
\text { diante transferencia } \\
\text { bancaria. }\end{array}$ & $\begin{array}{l}\text { Se trata de una ad- } \\
\text { quisición que lleva } \\
\text { IVA, además de un } \\
\text { pago por banco. } \\
\text { Junto con el IVA es } \\
\text { necesario explicar: } \\
\text {-Base Imponible } \\
\text {-Contrapartida } \\
\text {-Modelos } \\
\text {-Libros registro } \\
\text { factura emitida/ } \\
\text { recibida } \\
\text {-Utilidad del CIF }\end{array}$ & $\begin{array}{l}30 \\
\text { minutos }\end{array}$ \\
\hline 3 & $\begin{array}{l}\text { Ejercicio } \\
\text { práctico con } \\
\text { software }\end{array}$ & $\begin{array}{l}\text { Asiento manual por } \\
\text { compra de varios } \\
\text { con pago por caja }\end{array}$ & $\begin{array}{l}\text { Afianzar la mecá- } \\
\text { nica y aprender a re- } \\
\text { gistrar un pago por } \\
\text { caja. Configurar otros } \\
\text { modelos. }\end{array}$ & $\begin{array}{l}25 \\
\text { minutos }\end{array}$ \\
\hline 4 & $\begin{array}{l}\text { Ejercicio } \\
\text { práctico con } \\
\text { software }\end{array}$ & $\begin{array}{l}\text { Asiento con IVA } \\
\text { exento sin que se } \\
\text { registre pago }\end{array}$ & $\begin{array}{l}\text { Afianzar la mecánica } \\
\text { y comprender que } \\
\text { algunas activida- } \\
\text { des económicas es- } \\
\text { tán exentas de IVA. } \\
\text { No obstante, es ne- } \\
\text { cesario especificarlo. } \\
\text { Configurar este caso } \\
\text { particular. }\end{array}$ & $\begin{array}{l}25 \\
\text { minutos }\end{array}$ \\
\hline
\end{tabular}

Jornadas de Formación e Innovación Docente del Profesorado | № 1 (2018) Esta obra se distribuye con la licencia Creative Commons Reconocimiento-NoComercial-SinObraDerivada Internacional (CC BY-NC-ND 4.0.) 
Tabla 3. Secuencia de actividades de la tercera sesión

\begin{tabular}{|l|l|l|l|l|}
\hline & $\begin{array}{c}\text { Tipo de } \\
\text { actividad }\end{array}$ & \multicolumn{1}{|c|}{ Actividad } & Objetivos/Conceptos & \multicolumn{1}{c|}{$\begin{array}{c}\text { Tiempo } \\
\text { previsto }\end{array}$} \\
\hline 1 & $\begin{array}{l}\text { Narración } \\
\text { introductoria }\end{array}$ & $\begin{array}{l}\text { Conectar con la clase } \\
\text { anterior e introdu- } \\
\text { cir la temática de } \\
\text { la sesión: Asientos } \\
\text { predefinidos }\end{array}$ & $\begin{array}{l}\text { Reflexionar acerca de } \\
\text { las dificultades que } \\
\text { tiene la contabili- } \\
\text { dad manual frente a } \\
\text { la contabilidad auto- } \\
\text { mática desde varios } \\
\text { puntos de vista }\end{array}$ & mintos \\
\hline 2 & $\begin{array}{l}\text { Narración- } \\
\text { interactiva }\end{array}$ & $\begin{array}{l}\text { Aprender a pensar } \\
\text { como nuestro ERP } \\
\text { en cuanto a la in- } \\
\text { troducción de par- } \\
\text { tidas de Asientos } \\
\text { predefinidos }\end{array}$ & $\begin{array}{l}\text { Ver las diferentes } \\
\text { opciones de confi- } \\
\text { guración: partida, } \\
\text { subcuenta, importe, } \\
\text { concepto, contrapar- } \\
\text { tida, base imponible }\end{array}$ & 30 minutos \\
\hline 3 & $\begin{array}{l}\text { Ejercicio } \\
\text { práctico con } \\
\text { software }\end{array}$ & $\begin{array}{l}\text { Asiento predetermi- } \\
\text { nado de compras }\end{array}$ & $\begin{array}{l}\text { Practicar la mecánica } \\
\text { con una situación } \\
\text { habitual }\end{array}$ & 25 minutos \\
\hline 4 & $\begin{array}{l}\text { Ejercicio } \\
\text { práctico con } \\
\text { software }\end{array}$ & $\begin{array}{l}\text { Asiento predetermi- } \\
\text { nado de ventas }\end{array}$ & $\begin{array}{l}\text { Practicar la mecánica } \\
\text { con una situación } \\
\text { habitual, inten- } \\
\text { tando optimizar la } \\
\text { configuración }\end{array}$ & 25 minutos \\
\hline
\end{tabular}

\section{Seguimiento de la evolución de los modelos mentales de los estudiantes}

A este fin, se ha elaborado un cuestionario que recoge preguntas sobre los conceptos y problemas clave que se tratan en las sesiones del ciclo de mejora y que aparecen en el mapa de contenidos (en forma más escueta). Dicho cuestionario incluirá las mismas preguntas y se aplicará al inicio de la primera sesión y al final de la última. El objetivo principal de esta herramienta es evaluar, en términos generales, el aprendizaje del alumno y, además, conocer qué conceptos o problemas han sido más difíciles 
de interiorizar. El objetivo no es calificar al alumno, sino comprobar si ha habido un progreso en su conocimiento.

La evolución de su aprendizaje se obtendrá como resultado de comparar los conocimientos iniciales de los alumnos con los finales. Para ello, se usarán las denominadas escaleras de aprendizaje a nivel grupal con preguntas abiertas (con espacio limitado para su respuesta). En comparación a las cerradas, éstas aportan más información al docente sobre la lógica que siguen las respuestas y mayor fiabilidad (Porlán, 2017). De forma simplificada, la evaluación consiste en estudiar las respuestas de los alumnos y tratar de identificar los modelos de pensamiento que las sostienen, permitiéndonos agrupar las respuestas secuencialmente de modelos más simples a más complejos. Además de clasificar las respuestas de los alumnos y a su vez ordenarlas en grado de complejidad, también nos permitirá identificar los obstáculos que encuentran los alumnos al enfrentarse con la pregunta en cuestión, qué les puede estar frenando a la hora de dar respuestas más concretas. Si bien las escaleras de aprendizaje se pueden usar de forma grupal e individual, vamos a centrarnos en su uso colectivo. Esto ya supondrá una mejoría con respecto a la evaluación llevada a cabo en un ciclo anterior, donde se emplearon cuestionarios de preguntas cerradas (de tipo test) en las que se podía ver la evolución general pero no establecer grados de complejidad en los modelos de pensamiento de los alumnos.

Las preguntas que incluirá el cuestionario se recogen a continuación:

- Hasta ahora hemos estado trabajando con el módulo Área de Facturación en nuestro ERP, ¿hemos realizado alguna operación contable hasta el momento (ejemplo: registro de asientos contables? Justifica la respuesta.

Jornadas de Formación e Innovación Docente del Profesorado I № 1 (2018) Esta obra se distribuye con la licencia Creative Commons 
- En el Plan General de Contabilidad aparece desarrollado el Cuadro de Cuentas y en Aban-Q se cargó al instalar el programa, ¿crees que podemos modificarlo para ajustarlo a nuestra empresa o no sería correcto? Justifica la respuesta.

- Existen diferentes tipos de IVA, ¿sabrías enumerarlos y explicar brevemente por qué es necesaria cada tipología?

- ¿Qué utilidad puede tener la configuración de cuentas especiales en Aban-Q si se trata de un proceso interno del software?

- Si fueses el propietario, ¿cómo crees que podría afectarte el hecho de que tu empresa lleve parte de su contabilidad de manera automática? (por ejemplo, empleando asientos predefinidos).

\section{Evaluación del propio diseño y de la intervención del docente}

Se va a realizar mediante la elaboración de un "diario de clase" por parte del docente, donde anotar las reflexiones sobre cómo ha ido cada sesión, qué comportamientos hemos observado en nuestros alumnos, qué ha podido fallar, qué ha funcionado especialmente bien. Estas anotaciones servirán de apoyo para evaluar el diseño que se implementa en este curso y la propia intervención del docente, pero también como un documento al que acudir como punto de partida para el siguiente ciclo de mejora, intentando mejorar la calidad de los diseños e intervenciones de forma progresiva con decisiones más argumentadas, puesto que se basan en un documento y no sólo nuestros recuerdos (Porlán, 2017).

La progresión de los alumnos a la vista y análisis de las escaleras de aprendizaje, también se relacionarán con el éxito o fracaso del diseño del ciclo de mejora. El objetivo principal es incidir en el aprendizaje del alumno, por lo 
que una mejora o empeoramiento general en las respuestas incluidas en los cuestionarios debe relacionarse con el desempeño docente.

\section{Aplicación del Ciclo de Mejora Docente}

En términos generales, se ha podido aplicar el diseño del ciclo de forma fiel en su secuencia de actividades. Estimo que la decisión de no planificar el $100 \%$ del tiempo disponible de cada sesión ha sido un acierto, porque ha proporcionado margen para el inicio y cierre de sesión (gestionar la copia de seguridad) y la configuración y dinamización de los grupos de trabajo.

La segunda sesión ha constituido un reto mayor. Debido a que las sesiones del ciclo han coincidido en fecha con algunos exámenes parciales de la propia y de otras asignaturas, la afluencia a clase ha sido levemente inferior que en otros momentos. En concreto, coincidiendo con la segunda sesión hubo un volumen importante de alumnos que no habían participado en la primera, y no estaban familiarizados con la dinámica anterior. Aún así estimo que la respuesta general del grupo ha sido muy favorable.

\section{Evaluación del Ciclo de Mejora Docente. Conclusiones.}

Se ha efectuado el análisis pormenorizado de los cuestionarios iniciales y finales, y la clasificación de las respuestas de los alumnos en categorías presentadas en orden creciente de concreción y corrección de su modelo de pensamiento acerca del concepto evaluado (desde $\mathrm{A}=\mathrm{NS} / \mathrm{NC}$, hasta la letra que haya sido necesaria para clasificar las respuestas).

Analizando los resultados obtenidos, el avance más positivo ha tenido lugar en la asimilación del concepto Contabilidad Integrada (Muy Satisfactorio). Desaparecen 
las respuestas clasificadas como A, B, C en favor de la E, máxima categoría en este caso.

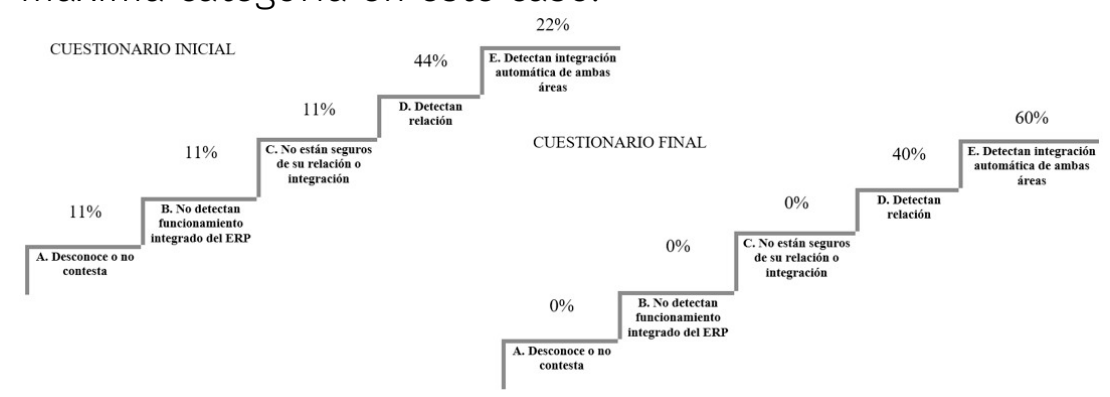

Figura 3. Escaleras de aprendizaje en una pregunta con evolución muy satisfactoria.

En el otro extremo de la balanza, el peor resultado es el correspondiente al concepto de Cuentas Especiales (No Satisfactorio). En este caso se observan leves bajadas de las categorías A, B y C en favor de la máxima, pero aún así las categorías quedan bastante equilibradas en número de respuestas, lo que denota que aún existe confusión en torno al concepto evaluado. Es posible que, en las sesiones posteriores al ciclo, cuando vean la utilidad real de la configuración de Cuentas especiales al elaborar los Modelos e Informes, entiendan mejor el proceso interno que ha seguido el software y su importancia.

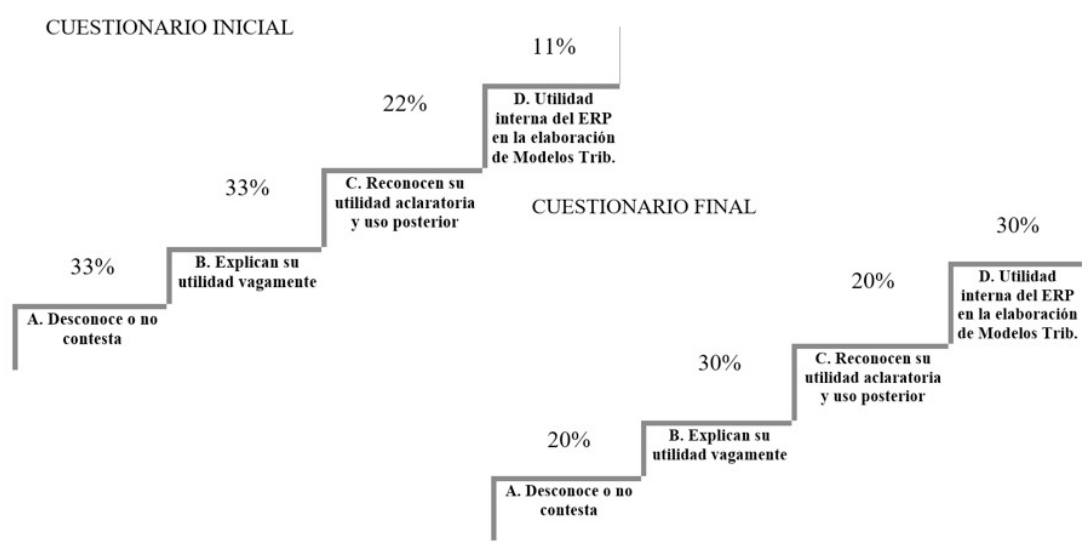

Figura 4. Escaleras de aprendizaje en una pregunta con evolución poco satisfactoria.

Jornadas de Formación e Innovación Docente del Profesorado | № 1 (2018) 
Las demás escaleras de conocimiento apuntan a una evolución satisfactoria, con cabida a mejorar, de los modelos de los alumnos. En la pregunta 2, Cuadro de Cuentas, la categoría $C$ ha disminuido (-26\%) en favor de la máxima, $\mathrm{E}$ (40\%, final). Para el caso del IVA, pregunta 4, las categorías $B$ y $C$ pierden respuestas en favor de otras más altas, $D$ y $E$ (30\% y 40\%, final), aunque la máxima, F, queda en un nivel similar (10\%). Por último, en la pregunta 5 , se constata que los efectos de la contabilidad automática para la empresa se perciben de forma positiva. En esta ocasión, la categoría B disminuye (-12\%) y la C desaparece (-22\%), aumentando el número de respuestas clasificadas en categorías más altas: D y E (porcentaje final de 60\% y $30 \%$, respectivamente). Se ilustra este último ejemplo, a modo de caracterización de este lote de preguntas con resultado satisfactorio en la evolución del aprendizaje.

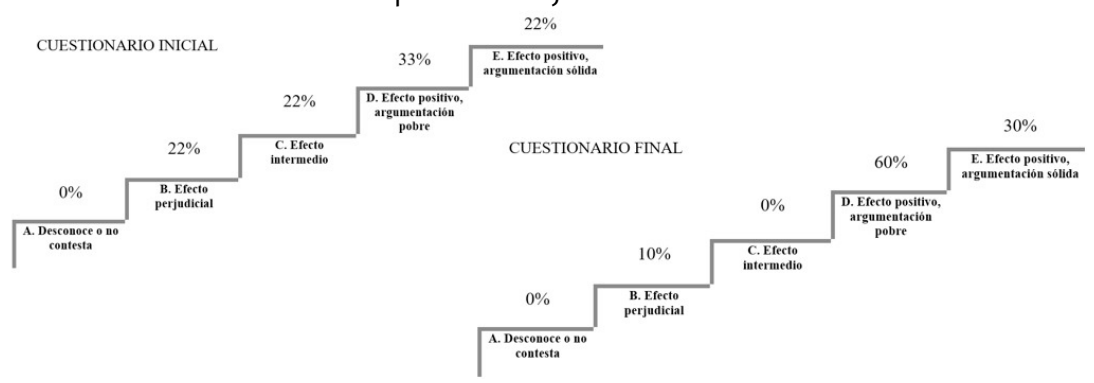

Figura 5. Escaleras de aprendizaje en una pregunta con evolución satisfactoria.

La segunda parte de la evaluación, la constituye el diario de clase elaborado por el docente. Estoy muy satisfecha con la respuesta del grupo, son alumnos con buena base de conocimientos y están comprometidos con sus estudios. Por mi parte, quisiera planificar el próximo ciclo asegurándome de que no coincide con períodos de exámenes, para que nos sea más sencillo mantener el enfoque durante su experimentación. También me gustaría mejorar dos aspectos claves de mi discurso: mayor precisión en los términos que empleo en mis explicaciones u orientaciones y planificación del total de los ejemplos que 
expongo en clase. Los ejemplos adicionales que no estén bien reflexionados en todos sus aspectos pueden restar en la compresión de conceptos, aunque la intención sea clarificar, incidir o dar una visión práctica.

Por último, quisiera seguir profundizando en la evaluación, intentando aplicar en el futuro cuestionarios iniciales y finales con alumnos identificados individualmente (de forma anónima mediante códigos) y aumentar la duración de los ciclos para que al menos abarquen una unidad completa y se terminen de establecer todas las relaciones de los conceptos implicados.

\section{Referencias bibliográficas}

Bain, K. (2007). Lo que hacen los mejores profesores universitarios. Valencia: Universitat de Valencia.

Finkel, D. (2005). Dar clase con la boca cerrada. Valencia: Universitat de Valencia.

Porlán, R. (2017). Enseñanza universitaria. Cómo mejorarla. Madrid: Ediciones Morata.

Sánchez-Lozano, G. (2016). Aplicación y análisis de cambios en la metodología de clases prácticas de la asignatura Sistemas de Información para las Finanzas y la Contabilidad. En R. Porlán, III Jornadas de Docencia Universitaria. Sevilla: Instituto de Ciencias de la Educación.

Jornadas de Formación e Innovación Docente del Profesorado I № 1 (2018) Esta obra se distribuye con la licencia Creative Commons 